\title{
Catheter related blood stream infections; the incidence and risk factors in Iranian hemodialysis patients
}

\author{
Amirhesam Alirezaei $^{1^{+}(\mathbb{C}}$, Nilofar Massoudi $^{2}$, Elham Zare ${ }^{3}$, Yasser Nouri ${ }^{3}$ \\ ${ }^{1}$ Urology and Nephrology Research Center, Shahid Modarres Hospital, Shahid-Beheshti University of Medical Sciences, Tehran, Iran \\ ${ }^{2}$ Critical Care Quality Improvement Research Center at Shahid Modarres Hospital, Department of Anaesthesiology, Shahid Beheshti \\ University of Medical Sciences, Tehran, Iran \\ ${ }^{3}$ Department of Internal Medicine, Shahid-Beheshti University of Medical Sciences, Tehran, Iran
}

\section{A R T I C L E I N F O}

\section{Article Type:}

Original

\section{Article History:}

Received: 17 March 2019

Accepted: 4 May 2019

Published online: 23 May 2019

\section{Keywords:}

Hemodialysis

Renal failure

Catheter-related infections

Staphylococcus

Renal failure

End-stage renal disease

\begin{abstract}
A B S T R A C T
Introduction: Patients with end-stage renal disease (ESRD) are routinely managed by hemodialysis. The catheter-related blood stream infections (CRBSIs) are important complications with high risk of mortality and morbidity in patients undergoing hemodialysis.

Objectives: To determine the incidence of CRBSIs in patients undergoing hemodialysis in a single center in Iran.

Patients and Methods: In this cross-sectional study, we evaluated the incidence of CRBSIs in 50 hemodialysis patients hospitalized at the Shahid-Modarres hospital of Tehran from March 2017 until March 2018. The data was collected from patients' medical archives. The CRBSI was established by blood culture.

Results: Of 50 hemodialysis patients enrolled in the study, 27 were males (54\%). The mean age was $49.7 \pm 11.8$ years old. Positive blood culture was observed in $64 \%$ of patients. The most common causative organism was coagulase-negative staphylococci (24\%). The most common comorbidity and systemic clinical symptom included hypertension (84\%) and fever (92\%) respectively. In most patients (74\%), the length of catheter indwelling was less than one year. Echocardiography showed that $24 \%$ of the patients had vegetation.

Conclusion: The coagulase-negative staphylococci were the most frequent organisms responsible for CRBSIs among hemodialysis patients. These findings might be helpful in early diagnosis and choose the best antibiotics for treatment.
\end{abstract}

\section{Implication for health policy/practice/research/medical education:}

To provide a guide on the timely use of appropriate antibiotics, we evaluated the incidence and risk factors of CRBSIs, as well as common causative microorganisms in hemodialysis patients. Based on our results coagulase-negative staphylococci are the most frequent organisms associated with CRBSI among hemodialysis patients in our hospital.

Please cite this paper as: Alirezaei AH, Massoudi N, Zare E, Nouri Y. Catheter related blood stream infections; the incidence and risk factors in Iranian hemodialysis patients. J Nephropharmacol. 2019;8(2):e17. DOI: 10.15171/npj.2019.17

\section{Introduction}

Renal failure is a common health problem worldwide. A large proportion of the health care budget is spent on managing patients with renal failure and end-stage renal disease (ESRD) by hemodialysis. Catheter-related blood stream infections (CRBSIs) are the leading complications in patients undergoing hemodialysis $(1,2)$. The CRBSIs lead to higher morbidity and mortality in hemodialysis patients and escalate health care costs, as well as hospitalization period (3-6). Because of the ease and quick accessibility, large vessels are commonly used during hemodialysis (7). As a result, these vessels (such as central venous) comprise common sources of CRBSIs.
Generally, the risk of all catheter-related infections (CRIs) and CRBSIs is higher in immunocompromised patients, those experiencing persistent catheter indwelling, and finally patients with history of CRIs (8). With the incidence ranging from $15 \%$ to $36 \%$, CRIs comprise the most common complications of indwelling catheters. The three common types of CRIs are the tunnel infection, exit site infection and CRBSIs. The most common location for CRIs is the site of catheter insertion $(9,10)$. The incidence of CRIs, and particularly sepsis, has been associated with 100 to 300 times, higher mortality rate in hemodialysis patients than general population (2). Cautions during catheter insertion, routine checking of the catheter 
entrance site (10-13), training of both patients and staff, hand washing and finally short-term application of catheters are essential factors decreasing the incidence of CRBSIs in dialysis patients (14-18).

The proportion of patients undergoing hemodialysis is rising in different populations. These patients experience frequent hospitalizations which impose high financial costs on the patients. As well, these patients are exposed to exaggerated risk of mortality and morbidity due to surgical procedures and frequent catheterization.

\section{Objectives}

To provide a guide on the timely administration of appropriate antibiotics, we here evaluated the incidence and risk factors of CRBSIs, as well as common causative microorganisms in hemodialysis patients referred to the Shahid-Modarres hospital of Tehran.

\section{Patients and Methods}

\section{Data collection}

This cross-sectional study was carried out on 50 hemodialysis patients admitted to the nephrology ward of Shahid-Modarres hospital in Tehran from March 2017 to March 2018. The patients with complete medical records were included. The history of CRBSIs was assessed by reviewing medical archives and interviewing the participants. The complementary data such as age, gender, duration of catheter indwelling, the duration of dialysis, type of vascular access, systemic clinical manifestations (fever and chills), purulent discharge and erythema at the catheterization exit site, endocarditis and finally hemodynamic instability was recorded by reviewing the clinical databases at the time of entry to the study.

Blood samples were taken to determine the type of organisms associated with CRBSIs. The diagnosis of CRBSIs was confirmed by blood culture test.

\section{Ethical issues}

The guidelines of the Declaration of Helsinki and its later amendments were followed. The design and objectives of the study were explained to all the participants, and written informed consent was obtained from them. This study was a result of a M.D, thesis of Yasser Nouri registered in Shahid Beheshti University of Medical Sciences (Thesis \# 223M).

\section{Statistical analysis}

The variables were described using either proportion and frequency (for qualitative variables) or mean and standard deviation (for quantitative variables). The student t-test (comparing the means of quantitative variables between study groups) and chi-square test (assessing associations between categorical variables) were used for inferential analyses. The statistical significance cut off was considered as $P$ value $<0.05$. The statistical procedures were performed in SPSS version 21 .

\section{Results}

Fifty hemodialysis patients admitted to the nephrology ward of Shahid- Modarres hospital (Tehran) with impression of CRBSI were assessed. Men and women comprised 27 (54\%) and $23(46 \%)$ of the participants respectively (Table 1 ). Males had higher risk of positive blood cultures $(P=0.041)$.

The mean age of the patients was $49.7 \pm 11.8$ years old. Considering different age groups, 2 (4\%), $6(12 \%), 8$ (16\%), 9 (18\%), $10(20 \%), 11(22 \%), 3(6 \%)$, and $1(2 \%)$ of the patients had 11-20, 21-30, 31-40, 41-50, 51-60, 61-70, 71-80 and 81-90 years old respectively.

In terms of systemic clinical signs, 46 (92\%) patients had fever and chills. Furthermore, 16 (32\%) and 11 (22\%) patients showed purulence and erythema at the site of catheter insertion respectively. The duration of catheter indwelling was $<1$ year in $37(74 \%)$ and $>1$ year in 13 (26\%) patients (Figure 1). Considering the comorbidities, hypertension, diabetes, and cardiovascular disease were observed in 42 (84\%), 26 (52\%), and 10 (20\%) patients respectively. In addition, 23 (46\%) patients had concurrent hypertension and diabetes.

The result of blood culture was positive in 32 (64\%) patients. The causative microorganisms included coagulase-negative staphylococci (12,24\%), Staphylococcus aureus (4, 8\%), Acinetobacter 4 (8\%), Klebsiella 3 (6\%), Pseudomonas 2 (4\%), E. coli 2 (4\%), and Enterobacter 1 (2\%) (Table 2).

The systolic blood pressure was measured as $<110 \mathrm{~mm}$ $\mathrm{Hg}, 110-140 \mathrm{~mm} \mathrm{Hg}, 140-170 \mathrm{~mm} \mathrm{Hg}$ and $>170 \mathrm{~mm}$ $\mathrm{Hg}$ in 8 (16\%), 15 (30\%), 23 (46\%), and $4(8 \%)$ patients respectively. The diastolic blood pressure was $<70 \mathrm{~mm} \mathrm{Hg}$,

Table 1. Age distribution and underlying diseases in dialysis patients diagnosed with central catheter related infection

\begin{tabular}{lll}
\hline Demographic characteristics & Number & Percent \\
\hline Age (year) & 2 & 4 \\
$<20$ & 6 & 12 \\
$21-30$ & 8 & 16 \\
$31-40$ & 9 & 18 \\
$41-50$ & 10 & 20 \\
$51-60$ & 11 & 22 \\
$61-70$ & 3 & 6 \\
$71-80$ & 1 & 2 \\
$81-90$ & & \\
Gender & 27 & 54 \\
Male & 23 & 46 \\
Female & & \\
Underlying disease & 42 & 84 \\
Hypertension & 26 & 52 \\
Diabetes & 23 & 46 \\
Hypertension and diabetes & 10 & 20 \\
Cardiovascular disease & & \\
Indwelling duration & 37 & 74 \\
$<1$ year & 13 & 26 \\
\hline 1 year & &
\end{tabular}




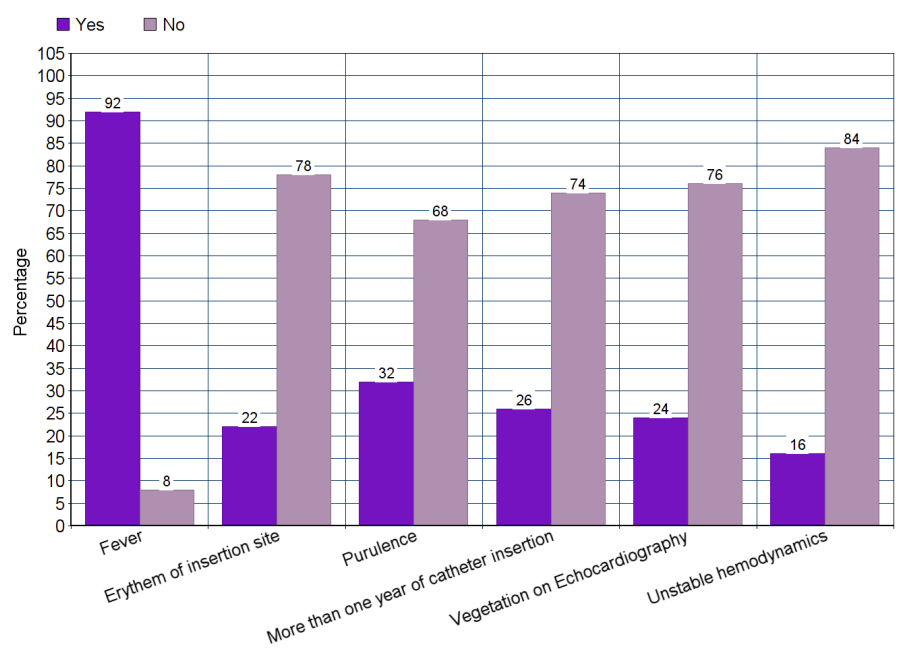

Figure 1. The frequency of systemic clinical signs, as well as infection signs at the site of catheter insertion in hemodialysis patients

$70-80 \mathrm{~mm} \mathrm{Hg}, 80-90 \mathrm{~mm} \mathrm{Hg}$ and $>90 \mathrm{~mm} \mathrm{Hg}$ in $2(4 \%)$, $13(26 \%), 19(38 \%)$, and $16(32 \%)$ patients respectively.

Significantly higher rate of CRBSIs was observed in patients belonged to more advanced age groups $(P=$ 0.04). No significant correlation was observed between the duration of indwelling and the incidence of CRBSIs $(P=0.09)$. However, there was a significant association between the presence of hypertension and the incidence of CRBSIs $(P=0.03)$.

\section{Discussion}

There is inadequate information about the health outcomes, the quality of care, and the causative microorganisms of CRBSIs in Iranian hemodialysis patients. Furthermore, the antimicrobial sensitivity of the microorganisms responsible for CRBSIs varies among different geographic regions. The incidence of CRBSIs dims the health outcomes in patients undergoing hemodialysis. The present study was carried out to assess the incidence of CRBSIs in patients undergoing hemodialysis in the nephrology ward of Shahid-Modarres hospital in Tehran, Iran.

Table 2. The frequency of organisms leading to catheter-related blood stream infection in patients under hemodialysis

\begin{tabular}{lll}
\hline The organisms & No. & Percent \\
\hline Coagulase negative staphylococci & 12 & 24 \\
Staphylococcus aureus & 8 & 16 \\
Acinetobacter & 4 & 8 \\
Klebsiella & 3 & 6 \\
Pseudomonas & 2 & 4 \\
Escherichia coli & 2 & 4 \\
Enterobacter & 1 & 2 \\
No growth & 18 & 36 \\
Total & 50 & 100 \\
\hline
\end{tabular}

In the current study, the prevalence of positive blood culture was significantly higher in males than females which was consistent with a previous report from Gilan, north of Iran (9). However, some other studies have reported no difference in the incidence of CRBSIs comparing males and females (2, 6-9). On the other hand, the incidence of CRIs has been higher in females than males in some other reports (2,14-16). Overall, our results asserted no significant association between gender and the risk of CRBSIs in patients undergoing hemodialysis.

According to our results, the highest incidence of CRBSIs was observed in patients with 61-70 years old. In line with our findings, patients $>70$ years old had also shown increased incidence of CRIs $(12,16)$. On the contrary, Hemmati et al (9) and Tokars et al (18) reported a decrease of about $60 \%$ in the incidence of CRIs with increasing age. In patients undergoing dialysis, no significant differences have been reported in the morbidity rate (19) and the risk of catheter-related bacteremia (20-22) at different age groups. Accessibility to vascular routes other than the central vein is much more difficult in patients with advanced age. Besides, long-term and recurrent application of vascular catheters is often associated with greater risk of failure in catheterization $(19,23,24)$. Hence, precise care of dialysis catheters is essential to ensure persistent vascular access in the patients.

In the current report, the most common clinical symptoms associated with catheterization were fever and chills. This was in accordance with the results of Hemmati et al (15). In another study performed in Mashhad, the most common symptoms were inflammation and tenderness at the site of catheter insertion (2). Our results indicated no significant relationship between the duration of indwelling and the risk of CRBSIs. This finding was also consistent with the results of Shahbazian et al (21) and Hadadzadeh et al (24). In another report in 2016, however, the duration of indwelling and the catheterization method 
were associated with the risk of CRIs in hemodialysis patients $(14,25)$.

In the current study, hypertension was the most common concomitant comorbidity. Nevertheless, this observation was not in agreement with the findings of Sani et al (2). Overall, hypertension, diabetes, atherosclerosis, and heart failure are the most common comorbidities observed in hemodialysis patients. In other studies, diabetes has been associated with a higher risk of CRBSIs in hemodialysis patients $(13,14,26)$. On the other hand, one study showed no relationship between the incidence of CRIs and diabetes (19).

Positive blood culture was observed in $64 \%$ of our patients. Coagulase-negative staphylococci were the most common bacteria identified in the current study. This result was consistent with the findings of Sani et al (2). In previous reports, $S$. aureus has been detected in about $64 \%$ of hemodialysis patients $(26,27)$. In one study, coagulasenegative staphylococci comprised the most prevalent organism with the prevalence of 50\% (28). Overall, our findings are in line with previous studies describing the distribution of CRBSIs causative microorganisms (2630 ). In the study of Almuneef et al, $48 \%$ of hemodialysis patients revealed a poly-microbial positive culture while $32 \%$ had grown gram-negative bacteria (28). Recent studies have declared an increment in the prevalence of gram-negative bacteria $(13,14,29)$.

The understanding of the epidemiology of CRBSIs causative microorganisms is important in terms of empirical antibiotic choice. Based on our observation, advanced age and hypertension were associated with increased risk of CRIs in patients undergoing hemodialysis. These findings can help to understand the microbial pattern of CRIs, to reduce the risk of CRIs, and to timely initiate appropriate antibiotic therapy.

\section{Conclusion}

Considering the limited studies on the incidence and risk factors of CRBSIs in Iranian hemodialysis patients, it is recommended to conduct more studies to address these issues. Furthermore, it is suggested to determine the microbial resistance pattern in these patients. Such information can assist nephrologists to undertake appropriate measures to prevent CRIs in hemodialysis patients.

\section{Limitations of the study}

One limitation of our study was that our results may not be generalizable to other nephrology centers in Iran because of low sample size. Also, the pattern of microbial resistance was not determined in the present study.

\section{Authors' contribution}

AA designed the study. YN performed the statistical analysis. EZ and NM drafted the manuscript. All the authors approved the final version of the manuscript.

\section{Conflicts of interest}

The authors declare that they have no competing interests.

\section{Ethical considerations}

Ethical issues (including plagiarism, data fabrication, double publication) have been completely observed by the authors.

\section{Funding/Support}

None.

\section{References}

1. Mozaffar M, Kharazm P, Delshad B, Majidi F. Permanent femoral hemodialysis catheters, better than reports: results of our technique in Shohadaye Tajrish Medical Center. Iran J Surg. 2016; 4:34-42.

2. 2.TavanaeeSani A, EslamiNokandeh A, Ghorbani $\mathrm{H}$. Study of central venous catheter -related infections in Hemodialysis patients. J Mashhad Univ Med Sci. 2012; 2:110-115. [Persian].

3. Chan KE, Maddux FW, Tolkoff-Rubin N, Karumanchi SA, Thadhani R, Hakim RM. Early outcomes among those initiating chronic dialysis in the United States. Clin J Am Soc Nephrol. 2011; 6:2642-9. doi:10.2215/CJN.03680411

4. Skov Dalgaard L, Nørgaard M, Jespersen B. Risk and prognosis of bloodstream infections among patients on chronic hemodialysis: a population-based cohort study. PLoS One. 2015;10:e0124547. doi:10.1371/journal. pone. 0124547

5. Goede MR, Coopersmith CM. Catheter-related bloodstream infection. Surg Clin North Am. 2009; 89:46374. doi:10.1016/j.suc.2008.09.003

6. Zhang $\mathrm{M}, \mathrm{Xu} \mathrm{Y}$, Jiang Z. Zhonghua Wei Zhong Bing Ji Jiu Yi Xue. 2017;29:1082-6. doi:10.3760/cma.j.is sn.2095-4352.2017.12.006

7. Daugirdas JT, Blake PG, Ing TS, eds. Handbook of Dialysis. 5th ed. Philadelphia, PA: Lippincott Williams \& Wilkins; 2014.

8. Seirafian S, Taheri S, Mortazavi M, Emami-Naeini A, Bahbouh WM. Determining and comparison of infectioncontrol status before and after educational intervention among patients and staff in hemodialysis ward. J Isfahan Med Sch. 2013; 31(237):675-83. [Persian].

9. Hemmati H, Safayiasl A, Badrangbuye S, Kazemnezhad Leyli E, Poursafar M, Jafaryparvar Z. Central venous catheter infections in hemodialysis patients. J Guilan Univ Med Sci 2018; 27:20-27. [Persian].

10. 10.Menegueti MG, Betoni NC, Bellissimo-Rodrigues F, Romão EA. Central venous catheter-related infections in patients receiving short-term hemodialysis therapy: incidence, associated factors, and microbiological aspects. Rev Soc Bras Med Trop. 2017;50:783-787. doi:10.1590/00378682-0438-2017

11. Shahidi SH, Soheilipour M. Comparison of vascular access use in hemodialysis patients in Isfahan in 2003 and 2013. Indian J Nephrol. 2015; 25:16-20. doi:10.4103/0971- 
4065.134656

12. Hussein WF, Mohammed H, Browne L, Plant L, Stack AG. Prevalence and correlates of central venous catheter use among haemodialysis patients in the Irish health system a national study. BMC Nephrol. 2018; 19:76. doi: 10.1186/ s12882-018-0873-x.

13. Sahli F, Feidjel R, Laalaoui R. Hemodialysis catheter-related infection: rates, risk factors and pathogens. J Infect Public Health. 2016; 10:403-8. doi:10.1016/j.jiph.2016.06.008

14. Hoen B, Kessler M. Risk factors for bacterial infections in chronic hemodialysis adult patients: a multicenter prospective survey. Nephrol Dal Transplant 1995;10:377381.

15. Ward DR, Moist LM, MacRae JM. Risk factors associated with hemodialysis central venous catheter malfunction; a retrospective analysis of a randomized controlled trial. Can J Kidney Health Dis. 2014;1:15. doi:10.1186/2054-3581-115

16. Murea M, James KM, Russell GB, Byrum GV 3rd, Yates JE, Tuttle NS, et al. Risk of catheter-related bloodstream infection in elderly patients on hemodialysis. Clin J Am Soc Nephrol. 2014; 9:764-770. doi: 10.2215/CJN.07710713.

17. Hoen B, Paul-Dauphin A, Hestin D, Kessler M. Epibacdial: a multicenter prospective study of risk factors for bacteremia in chronic hemodialysis patients. J Am Soc Nephrol. 1998;9:869-876.

18. Tokars JI, Light P, Anderson J, Miller ER, Parrish J, Armistead $\mathrm{N}$, et al. A prospective study of vascular access infections at seven outpatient hemodialysis centers. Am J Kidney Dis. 2001;37:1232-40. doi: 10.1053/ajkd.2001.24527.

19. Taylor G, Gravel D, Johnston L, Embil J, Holton D, Paton $\mathrm{S}$. Incidence of bloodstream infection in multicenter inception cohorts of hemodialysis patients. Am J Infect Control. 2004;32:155-60. doi: 10.1016/j.ajic.2003.05.007.

20. Mirko M, Xodo A, Alessi M. Elderly patient: which vascular access? Choice and management of vascular access in the elderly patient. Nephrol @ Point of Care. 2018. doi: $10.1177 / 2059300718755625$.

21. Shahbazian H, Hadadzade M. the impact of intraluminal vancomycin administration on prevention of hemodialysis catheter-related infections. J Kerman Univ Med Sci 2007; 14: 211-5. [Persian].

22. Gahlot R, Nigam C, Kumar V, Yadav G, Anupurba S. Catheter-related bloodstream infections. Int J Crit Illn Inj Sci. 2014;4:162-7. doi:10.4103/2229-5151.134184

23. Zhang G, Cortés-Penfield NW, Mandayam S. Dialysis Catheter-related bloodstream infections in patients receiving hemodialysis on an emergency-only basis: a retrospective cohort analysis. Clin Infect Dis. 2019;68:110116. doi:10.1093/cid/ciy555

24. Seifert H, Cornely O, Seggewiss K. Bloodstream infection in neutropenic cancer patients related to short-term nontunnelled catheters determined by quantitative blood cultures, differential time to positivity, and molecular epidemiological typing with pulsed-field gel electrophoresis. J Clin Microbiol. 2003;41:118-23. doi:10.1128/JCM.41.1.118-123.2003

25. Allon M. Treatment guidelines for dialysis catheter-related bacteremia: an update. Am J Kidney Dis. 2009;54:13-7. doi:10.1053/j.ajkd.2009.04.006

26. Ashby DR, Power A, Singh S. Bacteremia associated with tunneled hemodialysis catheters: outcome after attempted salvage. Clin J Am Soc Nephrol. 2009; 4:1601-5. doi:10.2215/CJN.01840309

27. Nassar GM, Ayus JC. Infectious complications of the hemodialysis access. Kidney Int. 2000;60:1-13. doi: 10.1046/j.1523-1755.2001.00765.x

28. Almuneef MA, Memish ZA, Balkhy $\mathrm{HH}$, Hijazi $\mathrm{O}$, Cunningham G, Francis C. Rate, risk factors and outcomes of catheter related bloodstream infection in a pediatric intensive care unit in Saudi Arabia. J Hosp Infect. 2006; 62:207-13. doi:10.1016/j.jhin.2005.06.032

29. Changing trends in antimicrobial resistance of major bacterial pathogens, 1985-2005: a study from a medical center in northern Taiwan. J Microbiol Immunol Infect. 2011;44:1318. doi: 10.1016/j.jmii.2010.02.004.

30. Lee SC, Chen KS, Tsai CJ. An outbreak of methicillinresistant Staphylococcus aureus infections related to central venous catheters for hemodialysis. Infect Control Hosp Epidemiol. 2004; 25:678-684. doi:10.1086/502462

Copyright $\odot 2019$ The Author(s); Published by Published by Society of Diabetic Nephropathy Prevention. This is an open-access article distributed under the terms of the Creative Commons Attribution License (http://creativecommons.org/licenses/by/4.0), which permits unrestricted use, distribution, and reproduction in any medium, provided the original work is properly cited. 